\title{
FLORA RIPARIA DE LOS RIOS SABINAS Y SAN RODRIGO, COAHUILA, MÉXICO
}

\author{
José Á. Villarreal Q. ${ }^{1}$, Miguel Á. Carranza P. ${ }^{1}$, Eduardo Estrada C. ${ }^{2}$ \\ Y ANDRÉs RODRíGUEZ G. ${ }^{1}$ \\ ${ }^{1}$ Universidad Autónoma Agraria Antonio Narro, \\ Departamento de Botánica, \\ 25315 Buenavista Saltillo, Coahuila, México \\ ${ }^{2}$ Universidad Autónoma de Nuevo León, \\ Facultad de Ciencias Forestales, \\ 67700 Linares, Nuevo León, México
}

\section{RESUMEN}

Se presenta un estudio florístico de las especies de la vegetación riparia de los ríos Sabinas y San Rodrigo del norte de Coahuila. Mediante recorridos en el área se colectó material que se herborizó para formar parte de la colección del herbario ANSM. Se identificaron 70 familias, 193 géneros y 243 especies. Las plantas arbóreas representan $9 \%$ del total, las arbustivas $11 \%$, las lianas $6 \%$, las herbáceas acuáticas y subacuáticas $21.4 \%$ y las herbáceas terrestres $52.6 \%$.

Palabras clave: Coahuila, flora riparia, México, vegetación.

\begin{abstract}
A floristic study of the riparian vegetation in the rivers Sabinas and San Rodrigo of northern Coahuila, Mexico, is presented. The area was explored and the botanical material herborized and deposited at the ANSM herbarium. A total of 70 families, 193 genera and 243 species were found. The trees in the flora are $9 \%$ of all the species, the shrubs $11 \%$, the vines $6 \%$, the aquatic and subaquatic herbs $21.4 \%$ and the terrestrial herbs $52.6 \%$.
\end{abstract}

Key words: Coahuila, Mexico, riparian flora, vegetation. 


\section{INTRODUCCIÓN}

Los ríos Sabinas y San Rodrigo se localizan en la parte nororiental del estado de Coahuila en la región hidrológica denominada Bravo-Conchos, ambos con dirección oeste-este en un intervalo altitudinal aproximado de 1000 a $300 \mathrm{~m}$, conformándose como tributarios del río Bravo. El Sabinas nace en la sierra Santa Rosa $\left(28^{\circ} 02^{\prime} \mathrm{N}, 101^{\circ} 48^{\prime} \mathrm{W}\right)$ con el nombre local de San Juan, se une al río Álamos cerca de Múzquiz, continuando con el nombre de Sabinas. Tiene aproximadamente

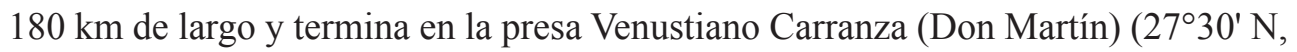
$100^{\circ} 43^{\prime} \mathrm{W}$ ), donde se une al Salado y continúa con ese nombre hacia el estado de Nuevo León. El trayecto incluye los municipios de Múzquiz, Sabinas y Juárez. El San Rodrigo se origina en las serranías del Burro ( $\left.28^{\circ} 55^{\prime} \mathrm{N}, 101^{\circ} 45^{\prime} \mathrm{W}\right)$, tiene cerca de $194 \mathrm{~km}$ de largo, cruza los municipios de Zaragoza y Piedras Negras y termina en el Bravo (28 $53^{\prime} \mathrm{N}, 100^{\circ} 40^{\prime} \mathrm{W}$ ) (Fig. 1).

Las cuencas de ambos ríos están comprendidas en el extremo noroccidental de la Planicie Costera del Golfo de México, con una topografía de lomeríos, donde los suelos son frecuentemente de tipo litosol y regosol y ocasionalmente xerosoles. La precipitación varía de los 350 a los $600 \mathrm{~mm}$ anuales y el clima es seco con una temperatura media anual de alrededor de $22^{\circ} \mathrm{C}$. La vegetación está formada por matorrales xerófilos donde es frecuente encontrar Leucophyllum frutescens (cenizo), Prosopis glandulosa (mezquite), Acacia rigidula (chaparro prieto), Castela erecta (chaparro amargoso) y Cercidium texanum (palo verde) entre otras. En los lomeríos de las serranías del Burro se presenta Agave lechuguilla (lechuguilla) y en los cañones de las sierras es común Acacia berlandieri (guajillo), Pithecellobium pallens (tenaza) y Colubrina greggii (manzanita) (Anónimo, 1983).

Se considera como vegetación riparia a aquella que crece a lo largo de corrientes de agua. Su delimitación generalmente es complicada, ya que además de las especies característicamente freáticas, es frecuente que muchas otras que crecen en comunidades vecinas también lo hagan en los márgenes de estos sistemas naturales de drenaje, creándose asociaciones vegetales que varían en densidad y diversidad florística (Dick-Peddie y Hubbard, 1977). Estas comunidades son de importancia ecológica, social y económica para la región, ya que constituyen el hábitat para la fauna silvestre, influyen en la calidad y cantidad de agua de los ríos, tienen uso recreativo, además de proporcionar forraje, leña y otros materiales (SolisG. y Jenkins, 1998).

La escasez de corrientes de agua permanentes en el interior del estado de Coahuila es notoria. Sólo algunos ríos del norte del estado mantienen un cauce de 


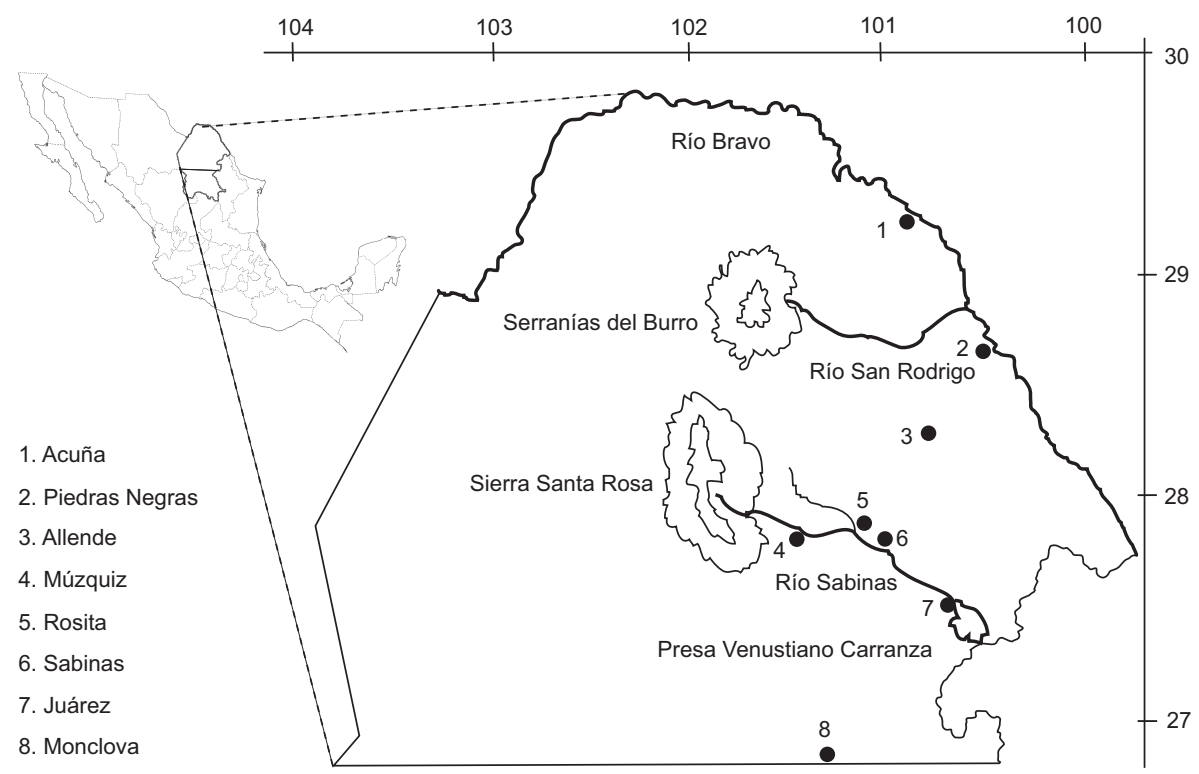

Fig. 1. Localización del área estudiada.

agua permanente o casi permanente durante todo el año y pueden sostener una vegetación riparia. Las comunidades que la forman son irregulares en su distribución y usualmente no figuran en los mapas de vegetación.

En la actualidad se llevan a cabo actividades que alteran el equilibrio de estas comunidades, como la extracción de materiales del suelo, el uso y retención de agua y la tala de árboles. Su fragilidad es muy evidente y su alteración tiene un efecto directo en las condiciones ecológicas del lugar.

En el presente estudio se proporciona un listado de las especies encontradas en la vegetación riparia de los ríos Sabinas y San Rodrigo, en el que se muestra su diversidad florística, con el propósito de contribuir a su mejor entendimiento, uso, manejo y conservación.

\section{MÉTODOS}

Se realizaron una serie de 12 recorridos en el área durante los años 1998-2002, con el propósito de inventariar los componentes de la vegetación riparia del norte del estado. Se llevaron a cabo colectas en forma selectiva, que permitieran incrementar 
la información florística y proceder a la toma de datos sobre el habitat y abundancia. El material (cerca de 850 muestras) se herborizó y determinó en el herbario ANSM, donde está depositado el juego principal de ejemplares. Con base en las características de los sitios en los cuales el material fue colectado, se reconocieron los siguientes hábitats: subacuático-acuático, ripario (vegetación asociada) y aledaño.

Se considera como parte del hábitat subacuático-acuático a las plantas que crecen en lugares inundados o dentro del agua. La vegetación asociada está formada por aquellos elementos que crecen a las orillas de corrientes de agua, comportándose como freáticas obligadas.

En la vegetación aledaña se incluye a los componentes de las comunidades de la vegetación dominante fuera del área riparia y que usualmente se presentan en los márgenes de los ríos; éstos frecuentemente se mezclan con la vegetación asociada. Son denominados especies riparias facultativas por Dick-Peddie y Hubbard (1977), o plantas "tolerantes" por Lot et al. (1998), ya que pueden quedar parcialmente sumergidas en lugares inundables durante periodos cortos.

La determinación se realizó con el uso de literatura taxonómica botánica y la nomenclatura de familias sigue la clasificación de Cronquist (1981) para magnoliofitas y a Crabbe et al. (1975) para helechos y grupos afines a helechos. Los nombres de los autores y abreviaciones siguen lo propuesto por Villaseñor (2001).

\section{RESULTADOS}

La flora riparia de los ríos Sabinas y San Rodrigo se encontró integrada por 243 especies, distribuidas en 193 géneros y 70 familias, como se muestra en el Cuadro 1. El número de arbóreas es 22 , lo que corresponde a $9 \%$ del total de las incluidas. Los arbustos son $26(11 \%)$, las lianas con tallos volubles o trepadores son $15(6 \%)$, las acuáticas y subacuáticas son $52(21.4 \%)$ y las hierbas de hábitat terrestre, también presentes en el área, son 129, lo que corresponde a 52.6\% del total de especies.

Los elementos de la comunidades de plantas asociadas son 117 especies, mientras que los componentes de las aledañas son 88 y los de ambiente subacuático y acuático 52 (Cuadro 2).

La composición florística de los bordes de los dos ríos es muy similar. En la vegetación riparia del Sabinas sobresale Taxodium mucronatum (sabino) como una especie común y dominante, que forma con frecuencia galerías estrechas a lo largo del río. Es frecuente encontrar también Fraxinus berlandieriana (fresno), Morus celtidifolia (mora), Celtis laevigata (palo blanco), Platanus glabrata (álamo) y Carya 
Cuadro 1. Participación cuantitativa de los grandes grupos de plantas vasculares en la flora riparia de los ríos Sabinas y San Rodrigo en Coahuila.

\begin{tabular}{|c|c|c|c|}
\hline Grupo Taxonómico & Familias & Géneros & Especies \\
\hline Pteridophyta & 4 & 8 & 10 \\
\hline Pinophyta & 1 & 1 & 1 \\
\hline Liliopsida & 8 & 35 & 51 \\
\hline Magnoliopsida & 57 & 149 & 181 \\
\hline Total & 70 & 193 & 243 \\
\hline
\end{tabular}

Cuadro 2. Participación cuantitativa de la flora riparia de los ríos Sabinas y San Rodrigo en Coahuila, en las diferente comunidades vegetales.

\begin{tabular}{|c|c|c|}
\hline Comunidades vegetales & Número de especies & Porcentaje \\
\hline Subacuática-acuática & 52 & 21.39 \\
\hline Aledaña & 88 & 36.2 \\
\hline Asociada & 117 & 48.15 \\
\hline
\end{tabular}

illinoinensis (nogal). En la parte baja, cercana a la presa Venustiano Carranza, Acacia farnesiana (huizache) es abundante.

Para el San Rodrigo las galerías se presentan discontinuas hasta llegar a elementos aislados; la especie más frecuente es Platanus glabrata (álamo) asociada con Carya illinoinensis (nogal), Fraxinus berlandieriana (fresno), Quercus fusiformis (encino), Morus celtidifolia (mora), Juglans microcarpa (nogalillo), Chilopsis linearis (mimbre) y ocasionalmente Prosopis glandulosa (mezquite). Las plantas arbustivas de estas comunidades son: Baccharis salicifolia y B. salicina (jarilla), Cephalanthus salicifolius (cabezona), Pluchea odorata (moradilla) y Arundo donax (carrizo). Estas comunidades son denominadas bosques de galería por Rzedowski (1978), o bosques subperennifolios riparios por Lot y Novelo (1990). 
Las asociaciones subacuáticas están dominadas por ciperáceas, algunas gramíneas y poligonáceas. Son comunes además: Bacopa monnieri, Cynoctomum mitreola, Justicia americana, Centella asiatica, Hydrocoyle spp., Conoclinium betonicifolium, Eclipta alba, Trichocoronis rivularis, Lobelia cardinalis, Lythrum californicum, Eustoma exaltatum, Samolus ebracteatus, Rorippa nasturtiumaquaticum y Typha latifolia. Las acuáticas se restringen a especies de los géneros: Ceratophyllum, Heteranthera, Potamogeton, Nymphaea, Nuphar y Utricularia. Es también común el alga Chara sp.

Ambos ríos están sujetos a disturbios que afectan el caudal de agua y su calidad. El más impactado parece ser el Sabinas que ha perdido o disminuido el flujo de agua en algunos lugares por la formación de excavaciones mineras (llamadas "tajos"), que al quedar a un nivel más bajo que su cauce, atrapan el agua que se filtra en el subsuelo.

En el apéndice se incluye un listado de las plantas encontradas en el área estudiada. Las categorías de familia, género y especie se presentan en secuencia alfabética. Se incluye el nombre científico de la especie, el tipo de vegetación en la que es frecuente, datos observados sobre su abundancia, el nombre del colector y el número de colecta.

\section{AGRADECIMIENTOS}

El presente trabajo se pudo llevar a cabo gracias al apoyo económico otorgado por la Universidad Autónoma Agraria Antonio Narro al proyecto "Flora riparia de los ríos Sabinas y San Rodrigo, Coahuila, México", con clave 020302052503.

\section{LITERATURA CITADA}

Anónimo. 1983. Síntesis geográfica de Coahuila, México. Instituto Nacional de Estadística, Geografía e Informática. Secretaría de Programación y Presupuesto. México, D.F. $163 \mathrm{pp}$.

Crabbe, J. A., A. C. Jermy y T. Mickel. 1975. A new generic sequence for the pteridophyte herbarium. Fern Gazette 11(2/3): 141-162.

Cronquist, A. 1981. An integrated system of classification of flowering plants. Columbia University Press. Nueva York. $1261 \mathrm{pp}$.

Dick-Peddie, W. A. y J. P. Hubbard. 1977. Classification of riparian vegetation. In: Johnson, R. R. y D. A. Jones (Tech. Coords.). Importance, preservation and management of 
riparian habitats: a symposium. USDA For. Ser. Gen. Tech. Rep. RM-43. Rocky Mt. For. and Range Exp. Stn. Ft. Collins, Colo. pp. 85-90.

Lot, A. y A. Novelo. 1990. Forested wetlands of Mexico. In: Lugo, A. E., M. M. Brison y S. Brown (eds.). Forested wetlands of the World. Vol. 15 Ecosystems of the World. Elsevier. Amsterdam. pp. 287-298.

Lot, A., A. Novelo y P. Ramírez. 1998. Diversidad de la flora acuática mexicana. In: Ramamoorthy, T. P. , R. Bye, A. Lot y J. Fa. (eds.). Diversidad biológica de México: orígenes y distribución. Instituto de Biología, Universidad Nacional Autónoma de México. México, D.F. pp. 563-578.

Martínez, M. y A. Novelo. 1993. La vegetación acuática del estado de Tamaulipas, México. Anales Inst. Biol. Univ. Nac. Autón. México, Ser. Bot. 64(2): 59-86.

Rzedowski, J. 1978. Vegetación de México. Ed. Limusa. México, D.F. 432 pp.

Solis-G., G. y P. Jenkins. 1998. Riparian vegetation on the río Santa Cruz, Sonora. USDA Forest Service Proceedings RMRS-P-5: 100-118.

Villaseñor, J. L. 2001. Catálogo de autores de plantas vasculares de México. Instituto de Biología, Universidad Nacional Autónoma de México y Comisión Nacional para el Conocimiento y Uso de la Biodiversidad. México, D.F. 40 pp.

Recibido en mayo de 2003.

Aceptado en diciembre de 2005. 
Apéndice. Lista de especies de plantas de la flora riparia de los ríos Sabinas y San Rodrigo. Abreviaciones de los colectores más frecuentemente citados: C (Miguel Á. Carranza P.), E (Juan A. Encina D.), Rdz (Andrés Rodríguez G.) y V (José Á. Villarreal Q.).

\section{PTERIDOPHYTA}

\section{EQUISETACEAE}

Equisetum laevigatum A. Braun. Subacuática-acuática. Escasa. C 3471

\section{POLYPODIACEAE}

Adiantum capillus-veneris L. Vegetación asociada. Común. C 3827, V 6089, 8906

Cheilanthes alabamensis (Bukley) Kuntze. Vegetación aledaña en cañones. Escasa. V 8924

Cheilanthes leucopoda Link. Vegetación aledaña en cañones. Rara. V 8926

Notholaena candida (M. Martens \& Galeotti) Hook. var. copelandii (Hall) Tryon. Vegetación aledaña en cañones. Escasa. V 8925

Pellaea sagittata (Cav.) Link var. cordata (Cav.) Tryon. Vegetación aledaña en cañones. Escasa. V 8927

Thelypteris ovata R. St. John var. lindheimeri (C. Chr.) A. R. Sm. Vegetación asociada. Común. C 2582, 3481, 3782, V 6082, 8912, 8928

\section{SCHIZAEACEAE}

Anemia mexicana Klotzsch var. mexicana. Vegetación aledaña en cañones. Común. V 8923, 8972

\section{SELAGINELLACEAE}

Selaginella lepidophylla (Hook. \& Grev.) Spring. Vegetación aledaña en cañones. Escasa. V 8929

Selaginella pilifera A. Br. Vegetación aledaña en cañones. Escasa. V 8942

\section{PINOPHYTA}

\section{TAXODIACEAE}

Taxodium mucronatum Ten. Vegetación asociada. Común. V 5996, 6003 
Apéndice. Continuación

\section{LILIOPSIDA (Monocolyledoneae)}

\section{AGAVACEAE}

Yucca rostrata Engelm. Vegetación aledaña. Escasa. C 2463

Yucca treculeana Carr. Vegetación aledaña. Escasa. C 2545

\section{ARACEAE}

Caladium bicolor (Aiton) Vent. Subacuática-acuática. Escasa. C 3493

\section{CYPERACEAE}

Cladium jamaicense Crantz. Subacuática-acuática. Escasa. C 2597, 3489, V 8946

Cyperus acuminatus Torr. \& Hook. Vegetación asociada y subacuática-acuática. Escasa. V 8889

Cyperus ochraceus Vahl. Subacuática-acuática. Escasa.V 6067, C 3350, 3464

Cyperus odoratus L. Vegetación asociada y subacuática-acuática. Común. C 2594, 3335, 3375, Rdz 1274, V 8890, 8899, 8979

Cyperus virens Michx. Subacuática-acuática. Escasa. V 8888, C 3463

Dichromena colorata (L.) Hitchc. Subacuática-acuática. Común. C 2578, 3487, Rdz 1063, 1281, V 5979, 8887

Eleocharis cellulosa Torr. Subacuática-acuática. Común. C 2595, E 1038, V 8885

Eleocharis geniculata (L.) Roem. \& Schult. Subacuática-acuática. Común. C 2577, 3334, 3458, E 1034, V 8898

Fuirena simplex Vahl. Subacuática-acuática. Común. C 2576, 3333, 3466, 3816, E 1036, V 5978, 8945, 8948, 8896

Rhynchospora harveyi W. Boott. Subacuática-acuática. Escasa. V 8944

Schoenoplectus americanus (Pers.) Volkard ex Schinz \& Keller. Subacuática-acuática. Escasa. C 3465

Schoenus nigricans L. Subacuática-acuática. Escasa. V 8983

\section{LILIACEAE}

Allium kunthii G. Don. Vegetación asociada. Escasa. C 3725

POACEAE

Andropogon glomeratus (Walter) Britton, Sterns \& Poggenb. Vegetación aledaña. Rara. C 3803 
Apéndice. Continuación

Arundo donax L. Vegetación asociada. Común. C 2598, 3477, 3805

Bouteloua curtipendula (Michx.) Torr. Vegetación aledaña. Común. C 3710

Bouteloua trifida S. Watson. Vegetación aledaña. Común. C 2449

Brachiaria fasciculata (Sw.) Parodi. Vegetación asociada. Escasa. V 6080

Cenchrus incertus M. A. Curtis. Vegetación asociada. Común. V 8956, C 3714

Chloris cucullata Bisch. Vegetación aledaña. Escasa. C 2451, 3097

Chloris subdolichostachya Mull. Berol. Vegetación asociada. Común. C 3090

Cynodon dactylon (L.) Pers. Vegetación asociada. Común. V 8539

Dichantium annulatum Stapf. Vegetación asociada. Escasa. C 2525, 3330, 3623

Digitaria cognata (Schult.) Pilg. Vegetación aledaña. Escasa. C 2518, 3716

Echinochloa colonum (L.) Link. Vegetación asociada y subacuática-acuática. Escasa. C 3777

Echinochloa walteri (Pursh) A. Heller. Subacuática-acuática. Escasa. C 3775

Elymus canadensis L. Vegetación asociada. Rara. C 3491

Eragrostis barrelieri Daveau. Vegetación aledaña. Común. C 2524

Eragrostis intermedia Hitchc. Vegetación aledaña. Común. C 3717

Eragrostis mexicana (Hornem.) Link ssp. mexicana. Vegetación aledaña. Escasa. C 3623

Hemarthria altissima (Poir.) Stapf. \& Hubb. Subacuática-acuática. Escasa. C 3102

Leersia monandra Sw. Vegetación asociada. Rara. V 8930

Panicum acuminatum Sw. Vegetación asociada. Común. C 2591, 3095, V 6077

Panicum hallii Vasey var. hallii. Vegetación aledaña. Común. C 2450, 2523, 3711, V 6031

Panicum virgatum L. Vegetación asociada. Escasa. C 3380, 3469, 3715, 3780, 3807

Paspalum pubiflorum Rupr. ex Fourn. var. pubiflorum. Subacuática-acuática. Común. C 3379, V 8985b

Setaria parviflora (Poir.) Kerguelen. Vegetación asociada. Común. C 2447, 3468

Setaria pumila (Poir.) Roem. \& Schult. Vegetación aledaña. Común. C 2446, 3332, 3719, 3806

Setaria scheelei (Steud.) Hitchc. Vegetación aledaña. Escasa. C 3378

Sorghum halepense (L.) Pers. Vegetación aledaña. Escasa. C 3804

Sporobolus cryptandrus (Torr.) A. Gray. Vegetación aledaña. Común. C 2452

Tridens albescens (Vasey) Wooton \& Standl. Vegetación asociada. Común. C 2448, 3712

Tridens muticus (Torr.) Nash. Vegetación aledaña. Común. C 3718

Tridens texanus (S. Watson) Nash. Vegetación aledaña. Común. C 3353, 3713

\section{POTAMOGETONACEAE}

Heteranthera dubia (Jacq.) MacMill. Subacuática-acuática. Escasa. C 3809

Stukenia striata (Ruiz \& Pav.) Holub. Subacuática-acuática. Escasa. C 3490

\section{SMILACACEAE}

Smilax bona-nox L. Vegetación asociada. Escasa. C 3784, Rdz 1258, V 6075 
Apéndice. Continuación

TYPHACEAE

Typha latifolia L. Subacuática-acuática. Escasa. C 3470

MAGNOLIOPSIDA (Dicotyledoneae)

\section{ACANTHACEAE}

Anisacanthus quadrifidus (Vahl) Nees var. wrightii (Torr.) Henr. Vegetación aledaña. Escasa. C 2566, 3518, 3731, Rdz 1053, 1264, V 8901

Justicia americana (L.) Vahl. Subacuática-acuática. Rara. C 2592, 3341, 3820, E 1044

Ruellia metzae Tharp. Vegetación aledaña. Escasa. C 3773

Siphonoglossa greggii Greenm. \& C. H. Thomps. Vegetación aledaña. Común. Rdz 951, V 6093

Tetramerium hispidum Nees. Vegetación asociada. Rara. Rdz 950

\section{ANACARDIACEAE}

Pistacia mexicana Kunth. Vegetación aledaña en cañones. Común. V 8938

Toxicodendron radicans (L.) Kuntze. Vegetación asociada. Escasa. C 2571, 2581, 3797, V 6000

\section{APIACEAE}

Centella asiatica (L.) Urb. Subacuática-acuática. Rara. C 2596, 3442

Cicuta maculata L. Subacuática-acuática. Rara. V 6074

Cyclospermum leptophyllum (Pers.) Sprague. Subacuática-acuática. Escasa. C 3337

Hydrocotyle umbellata L. Subacuática-acuática. Escasa. E 1041

Hydrocotyle verticillata Thunb. var. triradiata (Rich.) Fernald. Subacuática-acuática. Escasa. C 2575, 3818, V 5992

\section{ARISTOLOCHIACEAE}

Aristolochia coryi I. M. Johnst. Vegetación asociada. Rara. V6026

\section{ASCLEPIADACEAE}

Cynanchum unifarium (Scheele) Woodson. Vegetación asociada. Rara. C 2442, 2500 
Apéndice. Continuación

\section{ASTERACEAE}

Ageratina havanensis (Kunth) R. M. King \& H. Rob. Vegetación aledaña en cañones. Escasa. V 8936

Ambrosia confertiflora DC. Vegetación asociada. Común. V 8897

Ambrosia psilostachya DC. Vegetación asociada. Común. V 8960, C 2502, 3728

Ambrosia trifida L. Vegetación asociada. Común. C 3825, Rdz 1271

Aphanostephus ramosissimus DC. var. ramosissimus. Vegetación aledaña. Escasa. C 2440, 2527

Artemisia ludoviciana Nutt. Vegetación asociada. Rara. C 2555, 3432

Aster subulatus Michx. Vegetación asociada. Común. C 3795, Rdz 1256, V 8883

Baccharis salicifolia (Ruíz \& Pav.) Pers. Vegetación asociada. Común. C 2569

Baccharis salicina Torr. \& A. Gray. Vegetación asociada. Común. C 2568

Barroetea subuligera (Schauer) A. Gray. Vegetación asociada. Escasa. V 8952

Calyptocarpus vialis Less. Vegetación asociada. Común. Rdz 1245, C 3372

Chloracantha spinosa (Benth.) G. L. Nesom. Vegetación asociada. Común. V 8884, C 3431

Chromolaena odorata (L.) R. M. King \& H. Rob. Vegetación asociada. Escasa. C 3345

Cirsium texanum Buckley. Vegetación asociada. Común. C 2445, 3444

Conoclinium betonicifolium (Mill.) R. M. King \& H. Rob. Subacuática-acuática. Común. C 2587, 3524, 3798, E 1048, V8943

Conyza bonariensis (L.) Cronq. Vegetación asociada. Común. C 2476

Conyza canadensis (L.) Cronq. Vegetación asociada. Común. C 3456

Coreopsis cardaminaefolia (DC.) Nutt. Vegetación asociada y subacuática-acuática. Común. $\mathrm{V} 8980$

Eclipta alba (L.) Hassk. Subacuática-acuática. Rara. C 3811, V 6025

Eupatorium serotinum Michx. Vegetación asociada. Rara. C 3480, 3790, E 1051

Gnaphaliopsis micropoides DC. Vegetación aledaña. Común.V 8963

Helenium elegans DC. var. amphibolum Bierner. Vegetación asociada y subacuática-acuática. Rara. C 2457, 3482, V 5982, 8903

Helenium quadridentatum Labill. Vegetación asociada y subacuática-acuática. Rara. C 2490 Helianthus annuus L. Vegetación asociada. Escasa. C 2459, 3461

Heterotheca subaxillaris (Lam.) Britton \& Rusby. Vegetación asociada. Escasa. C 2468, 2556, 3781

Liatris punctata Hook. Vegetación asociada. Escasa. V 8907

Melampodium cinereum DC. var. hirtellum Stuessy. Vegetación aledaña. Común. V 8969

Palafoxia callosa (Nutt.) Torr. \& A. Gray Vegetación asociada y vegetación aledaña. Común. C 2476, 2539, 3356, 3447, V 8921, 8978

Parthenium hysterophorus L. Vegetación asociada. Común. C 3371

Pluchea odorata (L.) Cass. var. odorata. Vegetación asociada. Escasa. C 2455, 3476, E 1055 
Apéndice. Continuación

Porophyllum scoparium A. Gray. Vegetación asociada. Común. C 2484, 3516

Pyrrhopappus pauciflorus (Sessé \& Moc.) DC. Vegetación asociada. Rara. C 3381, 3788

Solidago velutina DC. Vegetación asociada. Escasa. Rdz 1269, V 8919

Spilanthes americana (Mutis) Hieron. Subacuática-acuática. Escasa. Rdz 1266

Thymophylla pentachaeta (DC.) Small. Vegetación asociada. Común. C 3373

Trichocoronis rivularis A. Gray. Subacuática-acuática. Escasa. V 6071, 8959, C 3346

Verbesina encelioides (Cav.) Benth. \& Hook. Vegetación asociada. Común. C 3374

Viguiera dentata (Cav.) Spreng. Vegetación asociada. Escasa. Rdz 1277, C 3433, 3500

\section{BIGNONIACEAE}

Campis radicans (L.) Seem. Vegetación asociada. Rara. V 5993, 6001

Chilopsis linearis (Cav.) Sweet var. linearis. Vegetación asociada. Escasa. C 2443, 3520

\section{BORAGINACEAE}

Heliotropium angiospermum Murr. Vegetación aledaña. Común. V5983, C 3344, 3377

Heliotropium confertifolium (Torr.) A. Gray. Vegetación aledaña. Escasa. C 2522

Heliotropium procumbens Mill. Vegetación asociada. Escasa. C 3338

Lithospermum matamorense A.DC. Vegetación asociada. Rara. Rdz 948, V 6068

\section{BRASSICACEAE}

Rorippa nasturtium-aquaticum (L.) Hayek. Subacuática-acuática. Escasa. C 3347

\section{CAMPANULACEAE}

Lobelia berlandieri A.DC. var. brachypoda (A. Gray) McVaugh. Vegetación asociada y subacuática-acuática. Escasa. C 2588, 3336, 3794, Rdz 949, V 5980

Lobelia cardinalis L. Subacuática-acuática. Escasa. C 2586, Rdz 1265, E 1047, V 5987

\section{CAPPARIDACEAE}

Polanisia uniglandulosa (Cav.) DC. Vegetación aledaña. Escasa. C 2485, 3723 Rdz 1056, 1262

\section{CAPRIFOLIACEAE}

Symphoricarpos orbiculatus Moench. Vegetación asociada. Escasa. Marsh 475 (TEX) 
Apéndice. Continuación

\section{CERATOPHYLLACEAE}

Ceratophyllum demersum L. Subacuática-acuática. Común. C 3735

\section{CHENOPODIACEAE}

Chenopodium ambrosioides L. Vegetación asociada. Común. C 3370

\section{CONVOLVULACEAE}

Evolvulus sericeus Sw. Vegetación aledaña. Escasa. V 8933

Ipomoea acuminata (Vahl) Roem. \& Schult. Vegetación aledaña. Escasa. C 2441, 3785

Operculina pinnatifida (Kunth) O’Donell. Vegetación asociada. Común. C 2521, 3517, Rdz 1267, V 6023

\section{CUCURBITACEAE}

Cucurbita foetidissima Kunth. Vegetación asociada. Escasa. C 3508

Melothria pendula L. Vegetación aledaña. Rara. C 3821

\section{EBENACEAE}

Diospyros texana Scheele. Vegetación aledaña. Escasa. C 2481

\section{EUPHORBIACEAE}

Acalypha hederacea Torr. Vegetación aledaña. Común. C 2472, 2560, Rdz 1054, V 8957

Croton fruticulosus Torr. Vegetación aledaña. Escasa. C 2482, 1552, E 1054

Croton monantogynus Michx. Vegetación asociada. Común. C 2458, 2488, 3514, E 1049, V 8965

Croton pottsii (K1.) Müll. Arg. Vegetación aledaña. Común. V 6066

Euphorbia cyathophora Murr. Vegetación aledaña en cañones. Común. V 8920, 8935

Euphorbia dentata Michx. Vegetación asociada. Común. C 2454

Euphorbia hyssopifolia L. Vegetación asociada. Común. Rdz 1044, 1242, C 3802

Euphorbia marginata Pursh. Vegetación asociada. Escasa. C 2589, 3519, Rdz 1241

Euphorbia serpens Kunth. Vegetación asociada. Común. Rdz 1244

Ricinus communis L. Vegetación asociada. Escasa. C 3436

Stillingia texana I. M. Johnst. Vegetación asociada. Común. C 2509, E 1064

Stillingia treculeana (Müll. Arg.) I. M. Johnst. Vegetación asociada. C 3446

Tragia ramosa Torr. Vegetación aledaña. Escasa. C 2531 
Apéndice. Continuación

\section{FABACEAE}

Acacia farnesiana (L.) Willd. Vegetación asociada-vegetación aledaña. Común. C 2541, $\mathrm{Rdz} 960$

Acacia greggii A. Gray var. wrightii (Benth.) Isely. Vegetación aledaña. Común. C 3363

Amorpha texana Buckley. Vegetación aledaña. Escasa. C 2565

Cercidium texanum A. Gray. Vegetación aledaña. Escasa. C 3454

Desmodium lindheimeri Vail. Vegetación asociada. Escasa. C 3342

Indigofera suffruticosa Mill. Vegetación asociada. Escasa. C 2562, Rdz 1059

Mimosa malacophylla A. Gray. Vegetación asociada. Común. C 2564

Mimosa turneri Barneby. Vegetación aledaña. Rara. C 3349

Neptunia pubescens Benth. Vegetación asociada. Rara. C 3455, 3801, 3812

Prosopis glandulosa Torr. var. glandulosa. Vegetación aledaña. Común. C 2543, 3362, 3440

Sesbania macrocarpa Muhl. Vegetación asociada. Escasa. V 8537, C 3441, 3789, 3829

\section{FAGACEAE}

Quercus fusiformis Small. Vegetación asociada. Común. C 2483, 2506, 2553, E, 1057, 1062, Rdz 954, V 8971

\section{GENTIANACEAE}

Centaurium calycosum (Buckley) Fernald var. calycosum. Vegetación aledaña. Escasa. C 2593, V 5991

Eustoma exaltatum (L.) G. Don. Subacuática-acuática. Común. C 2579, 3730, E 1045, Rdz 1060

\section{HYDROPHYLLACEAE}

Nama jamaicense L. Vegetación asociada. Escasa. V 8962

\section{JUGLANDACEAE}

Carya illinoinensis (Wang.) K. Koch. Vegetación asociada. Común. C 2510, 3435, 3449, 3819, E 1058, 1062, V 6076

Juglans microcarpa Berl. Vegetación asociada. Común. C 2511, 3448, 3729, Rdz 1064, V 5994

\section{LAMIACEAE}

Hedeoma drummondii Benth. Vegetación aledaña. Común. C 2512, 3522, 3793, Rdz 1050 
Apéndice. Continuación

Physostegia virginiana (L.) Benth. ssp. praemorsa (Shinners) Cantino. Vegetación asociada y subacuática-acuática. Escasa. C 2549, 2572, 3492, Rdz 1058

Poliomintha glabrescens A. Gray. Vegetación asociada. Escasa. C 2557, V 8974

Salvia ballotaeflora Benth. Vegetación aledaña. Común. C 3339, 3357

Salvia roemeriana Scheele. Vegetación asociada. Escasa. C 2487, 3512, 3722, 3783

Teucrium cubense Jacq. Vegetación asociada. Común. V 5989

\section{LENTIBULARIACEAE}

Utricularia vulgaris L. Subacuática-acuática. Escasa. C 2583, 3452, V 8895

\section{LOASACEAE}

Cevallia sinuata Lag. Vegetación aledaña. Común. C 2526

Eucnide bartonioides Zucc. Vegetación aledaña en cañones. Escasa. V 8917

\section{LOGANIACEAE}

Cynoctomum mitreola (L.) Britton. Vegetación asociada y subacuática-acuática. Común. C 2584, E 1043, V 5997, 8894, 8934, 8975

\section{LYTHRACEAE}

Cuphea viscosissima Jacq. Vegetación asociada. Escasa. V 6024

Lythrum californicum Torr. \& A. Gray. Vegetación asociada y subacuática-acuática. Común. V 5981, 8967, 8961, C 2456, 3459

\section{MALPIGHIACEAE}

Thryallis angustifolia (Benth.) Kunth. Vegetación aledaña. Escasa. C 3733

\section{MALVACEAE}

Abutilon fruticosum Gillies \& Perr. Vegetación aledaña. Escasa. Rdz 1249, V 6092 Abutilon hypoleucum A. Gray. Vegetación aledaña. Escasa. V 8916 Allowissadula holosericea (Scheele) Garcke. Vegetación aledaña. Escasa. E 1052 Herissantia crispa (L.) Brizicky. Vegetación aledaña. Común. C3348 Malvastrum coromandelianum (L.) Garcke. Vegetación asociada. Escasa. Rdz 1247 
Apéndice. Continuación

Sida abutifolia Mill. Vegetación asociada. Escasa. Rdz 1248

Sida tragiifolia A. Gray. Vegetación asociada. Común. V 6073, 8971

Wissadula amplissima (L.) Fr. Vegetación asociada. Escasa. C 3340

\section{MORACEAE}

Morus celtidifolia Kunth. Vegetación asociada. Común. C 3473, 2512, 3438, 3786, 3814

\section{NYCTAGINACEAE}

Boerhavia linearifolia A. Gray. Vegetación aledaña. Escasa. C 3724

\section{NYMPHAEACEAE}

Nuphar advena (Aiton) W. T Aiton. Subacuática-acuática. Escasa. C 3815, E 1056

Nymphaea ampla (Salisb.) DC. Subacuática-acuática. Escasa. C 3365 (río San Diego)

\section{OLEACEAE}

Fraxinus berlandieriana A. DC. Vegetación asociada. Común. C 2567, V 8947

Fraxinus greggii A. Gray. Vegetación aledaña. Común. C 2546

\section{ONAGRACEAE}

Calylophus berlandieri Spach. Vegetación aledaña. Escasa. C 2467, Rdz 1062

Gaura calcicola Raven \& Greg. Vegetación aledaña. Escasa. C 2466

Gaura parviflora Hook. Vegetación asociada. Escasa. Rdz 1061

Ludwigia octovalvis (Jacq.) Raven. Subacuática-acuática. Común. C 2551, 3475, 3824, E 1040, V 5988, 8973

\section{PAPAVERACEAE}

Argemone sanguinea Greene. Vegetación asociada. Común. C 3474

\section{PASSIFLORACEAE}

Passiflora foetida L. var. gossypiifolia (Ham.) Mast. Vegetación aledaña. Escasa. C 3727, E 1060, Rdz 1261, V 8964

Passiflora tenuiloba Engelm. Vegetación aledaña. Rara. C 3369, V 8913 
Apéndice. Continuación

PHYTOLACCACEAE

Phytolacca octandra L. Vegetación aledaña. Rara. C 3434

Rivina humilis L. Vegetación aledaña. Común. Rdz 1272

PLANTAGINACEAE

Plantago major L. Vegetación asociada. Rara. C 3428

PLATANACEAE

Platanus glabrata Fernald. Vegetación asociada. Común. C 2460, 3445, 3796, Rdz 1052, V 6002

\section{POLEMONIACEAE}

Gilia incisa Benth. Vegetación aledaña. Escasa. C 2471, Rdz 1041

Gilia rigidula Benth. Vegetación aledaña. Escasa. C 3472

\section{POLYGONACEAE}

Polygonum punctatum Elliott. Subacuática-acuática. Común. Rdz 1040, V 8882

Polygonum hydropiperoides Michx. Subacuática-acuática. Escasa. C 3822

\section{PRIMULACEAE}

Samolus ebracteatus Kunth var. cuneatus (Small) Henr. Subacuática-acuática. Común. C 2599, 3343, 3453, 3808, E 1042, V 6090, 8953

\section{RANUNCULACEAE}

Clematis pitcheri Torr. \& A. Gray. Vegetación aledaña. Rara. C 3823

\section{RHAMNACEAE}

Colubrina greggii S. Watson. Vegetación aledaña y vegetación asociada. Común. Rdz 1278, V 5986

Colubrina texensis (Torr. \& A. Gray) A. Gray. Vegetación aledaña. Escasa. C 2554, 3366, 3800

Condalia viridis I. M. Johnst. Vegetación aledaña. Escasa. C 2520 
Apéndice. Continuación

Ziziphus obtusifolia (Torr. \& A. Gray) A. Gray. Vegetación aledaña. Común. C 3450

ROSACEAE

Rubus trivialis Michx. Vegetación asociada. Escasa. V 6091, C 3503

RUBIACEAE

Cephalanthus salicifolius Humb. \& Bonpl. Vegetación asociada. Común. C 2580, 3488, 3799, E 1033, Rdz 1065, V 5995, 8886

Hedyotis nigricans (Lam.) Fosberg. Vegetación aledaña. Común. C 2489, 2515, Rdz 1051

\section{RUTACEAE}

Amyris madrensis S. Watson. Vegetación aledaña en cañones. Escasa. V 8914, 8940

Zanthoxylon hirsutum Buckley. Vegetación aledaña. Rara. C 2507, 2535

Zanthoxylon fagara (L.) Sarg. Vegetación aledaña. Común. V 8915

\section{SAPINDACEAE}

Ungnadia speciosa Endl. Vegetación asociada. Escasa. C 2444

\section{SCROPHULARIACEAE}

Bacopa monnieri (L.) Wettst. Subacuática-acuática. Escasa. C 2585, 3485, 3787, E 1046, Rdz 1255

Lamourouxia dasyantha (Cham. \& Schltdl.) Ernst. Vegetación asociada. Rara. V 5984

Leucophyllum frutescens (Berl.) I. M. Johnst. Vegetación aledaña. Común. C2547, 3354, 3511 Maurandya antirrhiniflora Willd. ssp. antirrhiniflora. Vegetación asociada. Escasa. C 3720 Mecardonia vandellioides (Kunth) Pennell. Vegetación asociada y subacuática-acuática. Escasa. C 2461

\section{SOLANACEAE}

Chamaesaracha sordida (Dunal) A. Gray. Vegetación aledaña. Escasa. C 2469

Datura wrightii Regel. Vegetación asociada. Escasa. C 2514

Nicotiana repanda Willd. Vegetación asociada. Escasa.V 6065

Nicotiana trigonophylla Dunal. Vegetación asociada. Común. C 2464, V 8949

Physalis viscosa L. Vegetación aledaña. Común. C 2559

Quincula lobata (Torr.) Raf. Vegetación aledaña. Escasa. C 3376 
Apéndice. Continuación

Solanum nigrum L. Vegetación asociada. Común. Rdz 1064, V 5998

STERCULIACEAE

Melochia pyramidata L. Vegetación asociada. Escasa. C 2590, 3732, 3826, Rdz 1263

ULMACEAE

Celtis laevigata Willd. Vegetación asociada. Escasa. C 2501, 2528, 3429

Celtis pallida Torr. Vegetación aledaña. Común. C 3367, 3430, Rdz 963

Celtis reticulata Torr. Vegetación asociada. Rara. C 3473, E 1063

Ulmus crassifolia Nutt. Vegetación asociada en arroyo Las Vacas. Rara. E 1031, 1059

\section{URTICACEAE}

Boemeria cylindrica Sw. Vegetación asociada. Rara. V 6004, 6670, C 3526

\section{VERBENACEAE}

Lantana horrida Kunth. Vegetación aledaña. Escasa. C 2465, 3355, 3721, 3792, Rdz 1254

Lantana macropoda Torr. Vegetación aledaña. Común. C 3791

Phyla nodiflora (L.) Greene var. incisa (Small) Moldenke. Vegetación asociada y subacuáticaacuática. Común. C 2508, 2570, 3443, 3726, E 1039, V 8900

Verbena neomexicana (A. Gray) Small. Vegetación asociada. Escasa. Rdz 1253

Verbena scabra Vahl. Vegetación asociada. Escasa. C 3460

\section{VITACEAE}

Vitis cinerea Engelm. Vegetación asociada. Escasa. C 2529, 2550, 3810, E 1032, Rdz 1270, V 6069, 8891

\section{ZYGOPHYLLACEAE}

Guaiacum angustifolium Engelm. Vegetación aledaña. Común. C 3368 